\title{
Development and Application of Terahertz Pulsed Imaging for Nondestructive Inspection of Pharmaceutical Tablet
}

\author{
Yao-Chun Shen and Philip F. Taday
}

(Invited Paper)

\begin{abstract}
Terahertz pulsed imaging (TPI) was evaluated for nondestructively characterizing the 3-D internal structures of pharmaceutical tablets. The structural information of a pharmaceutical tablet, such as coating thickness and interface uniformity, was obtained directly from the analysis of the time-domain terahertz waveform. The chemical map of a sample was obtained by using frequency-domain terahertz spectra, together with spectral matching techniques such as cosine correlation mapping. The axial spatial resolution achieved was $30 \mu \mathrm{m}$, limited by the confinement of terahertz pulses in time (pulse width); and the lateral spatial resolution was determined to be $150 \mu \mathrm{m}$ at $90 \mathrm{~cm}^{-1}(2.7 \mathrm{THz})$, limited by the confinement of terahertz pulses in space (focus size, which is diffraction limited and thus frequency dependent). In addition, the buried structure within a tablet was mapped using the TPI, and its chemical composition was successfully identified through spectral-time analysis of the recorded terahertz waveform. We also present a rigorous electromagnetic theory for simulating the terahertz propagation in a multilayered sample, to facilitate terahertz data analysis and interpretation. In conclusion, the TPI is a powerful tool for assaying the tablet coating layer thickness and interface uniformity, and for identifying polymorphs.
\end{abstract}

Index Terms-Chemical mapping, multilayer coatings, pharmaceutical tablet, structural mapping, terahertz pulsed imaging (TPI).

\section{INTRODUCTION}

I $\mathrm{N}$ THE pharmaceutical sector, there is an increasing trend toward the development of solid dosage forms with more sophisticated coatings or inner structures. These functional internal structures provide essential mechanisms for controlling the release kinetics of the active pharmaceutical ingredient (API) and/or for anticounterfeit purpose [1], [2]. For example, coatings perform a wide variety of functions, and pharmaceutical tablet coatings are actually now one of the preferred routes to control the release of active drug ingredients in the body. If the coating is nonuniform or has defects, then the desired dose delivery and bioavailability can be compromised. Therefore, there is a critical need of new techniques for mapping and characterizing these internal structures nondestructively. Various imaging

Manuscript received October 9, 2007; revised September 10, 2007.

Y.-C. Shen is with the Department of Electrical Engineering and Electronics, University of Liverpool, Liverpool L69 3GJ, U.K. (e-mail: y.c.shen@liv.ac.uk).

P. F. Taday is with the TeraView Ltd., Cambridge CB4 0WS, U.K. (e-mail: phil.taday@teraview.com).

Color versions of one or more of the figures in this paper are available online at http://ieeexplore.ieee.org.

Digital Object Identifier 10.1109/.2007.JSTQE.911309 methods have been proposed to assay the product performance through evaluating the physical and chemical uniformity inside a tablet, and some yield impressive results [3]-[6]. Infrared (IR) and/or Fourier transform IR (FTIR)-Raman imaging provides information on the uniformity of active ingredients in a sample (which is near or at the surface). A full 3-D chemical map would, however, require slicing through the tablet many times, and this is destructive, time consuming, and may cause changes to the tablet under study. On the other hand, X-ray computed tomography provides full 3-D maps of density uniformity of a tablet, but it has little or no chemical specificity to differentiate, for example, the active ingredient from excipients of a tablet. There is also a safety concern about using ionizing $X$-ray radiations.

The terahertz region of the electromagnetic spectrum ( $40 \mathrm{GHz}-4 \mathrm{THz}, 1.33-133 \mathrm{~cm}^{-1}$ ) has a unique combination of properties in that terahertz waves can propagate through most pharmaceutical materials; many excipients and active ingredients show characteristic spectral features in the terahertz region; and the radiation is nonionizing and is safe to use [7], [8]. We have previously demonstrated that terahertz pulsed imaging (TPI) can be used for nondestructive analysis of coated tablets [9]-[12]. In this paper, we demonstrate further that the buried structure within a pharmaceutical tablet can be mapped nondestructively using the TPI technique. Furthermore, the chemical identity of the buried structure can also be revealed by using its spectral signatures. We will provide experimental results on the axial and lateral spatial resolution of the TPI maps and discuss application of terahertz imaging for 3-D chemical mapping.

\section{EXPERIMENTAL METHOD}

In all measurements, we used a TPI imaga 2000 system (TeraView Ltd, U.K.). This has been purposely developed for the fully automated scan of typical pharmaceutical solid dosage forms. In brief, terahertz radiation is generated by pumping a biased photoconductive antenna with an ultrashort laser pulse from a Ti:Sapphire laser (Fig. 1). The emitted terahertz pulse is collected, collimated, and then focused onto a sample under test. The reflected and backscattered terahertz pulse is then collected and focused onto an unbiased photoconductive antenna for the laser-gated terahertz detection [13], [14]. This coherent field detection scheme not only yields terahertz spectra with excellent SNR and high dynamic range, but also allows both the absorption coefficient and refractive index to be determined without the need to apply the Kramers-Kronig dispersion relationship. 


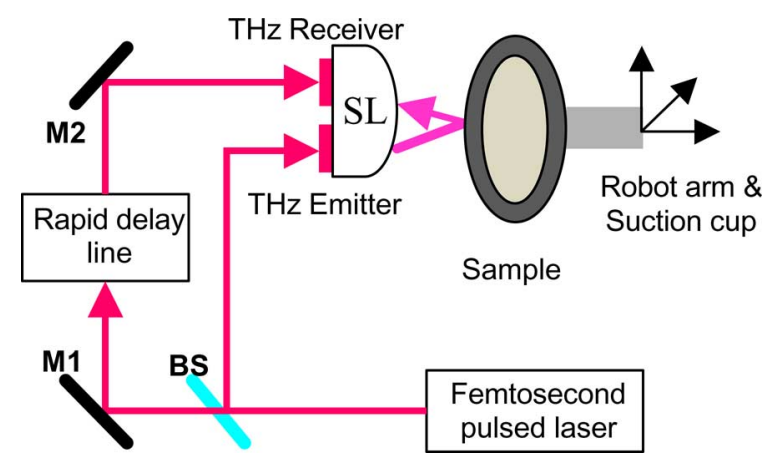

Fig. 1. Schematic diagram of a TPI system. A six-axis robot system was used to handle the sample. SL: Silicon lens system; BS: Beam splitter; M1 and M2: Metallic mirrors.

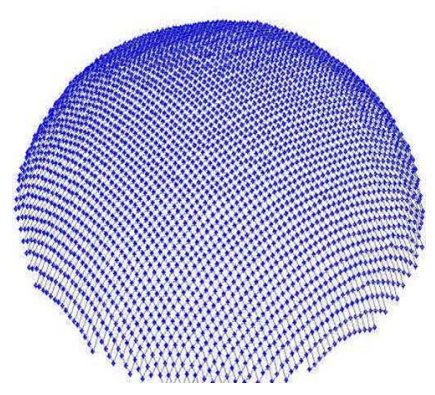

Fig. 2. Surface model of an M\&M tablet obtained using a laser gauge scan.

In addition, both the terahertz emitter and detector were directly attached to a specially designed silicon lens system, thus avoiding the requirement of nitrogen purging [water vapor absorption was minimized owing to the shorter distance $(8 \mathrm{~mm})$ between the terahertz lens and the sample].

Most pharmaceutical tablets have curved surface; therefore, a laser gauge employing a visible laser beam with a wavelength of $670 \mathrm{~nm}$ was used to precisely model the surface shape/curvature of the tablet under investigation. The generated surface model was subsequently used for TPI measurement, thus ensuring that during TPI measurement, the sample is always at the terahertz focus position with the sample surface always perpendicular to the terahertz probe (a six-axis robot system was introduced to handle the sample). Fig. 2 shows an example of such a model obtained from an M\&M chocolate, where solid circles represent terahertz measurement points.

For all measurements reported here, the TPI imaga 2000 system was operating at a rapid scan mode, i.e., at 30 complete waveforms/s. At each pixel, the terahertz radiation reflected from a sample was recorded as a function of time over a scan range of $2 \mathrm{~mm}$ for coating analysis, and $5 \mathrm{~mm}$ for chemical mapping. A 5-mm scan range allows mapping deeper buried structures inside a tablet, and it also provides a better spectral resolution of $1 \mathrm{~cm}^{-1}(0.03 \mathrm{THz})$ in the spectral range 5$100 \mathrm{~cm}^{-1}(0.15-3 \mathrm{THz})$. A full terahertz scan will eventually cover the entire surface of the tablet, thus producing a 3-D TPI data for each sample scanned. Usually, a metallic mirror is used as a reference, and the recorded reference and sample waveforms are then used for the reconstruction of a 3-D terahertz map.

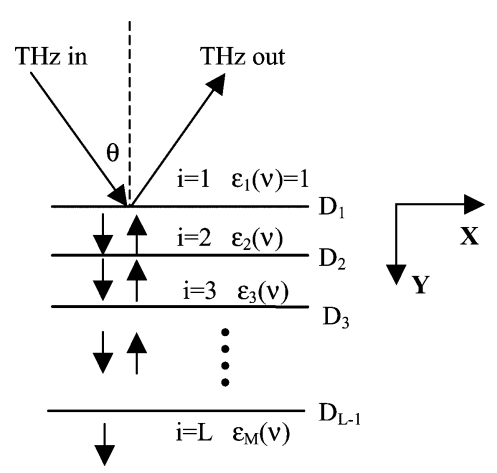

Fig. 3. 1-D model for simulating terahertz propagation in a multilayered sample.

\section{DATA ANALYSIS}

\section{A. Terahertz Propagation in a Multilayered Medium}

In order to analyze and interpret terahertz data, we describe in this section a rigorous electromagnetic theory for studying the terahertz propagation in a multilayered medium. The timedomain terahertz incident pulse was first Fourier transformed to frequency domain. The transmission and reflection coefficients were then calculated at each frequency using a 1-D model [15], together with a Lorentz model to simulate the complex dielectric function for each layer of the sample. The calculated reflection coefficients were finally Fourier transformed back to time domain to obtain the reflected terahertz waveform. Fig. 3 shows the 1-D model, where the sample is uniform in the transverse and has L-layers in depth direction. For simplicity, we assume that the incident terahertz radiation is $p$-polarized (the $s$-polarized case can be analyzed in a similar manner). Thus, the magnetic field of the terahertz radiation only has a $z$-component, which can be expressed as [15]

$$
H_{z}^{(i)}(x, y)=A_{i} \exp \left(j\left(\beta_{0} x-\beta_{i} y\right)\right) \quad(i=1,2, \ldots, L)
$$

where

$$
\begin{aligned}
& \beta_{0}=\sqrt{\varepsilon_{1}(v)} k_{0} \sin \theta \\
& \beta_{i}=\sqrt{\varepsilon_{i}(v) k_{0}^{2}-\beta_{0}^{2}} \quad(i=1,2, \ldots, L)
\end{aligned}
$$

with $k_{0}$ being the wavevector in vacuum, $\theta$ the incident angle, and $\varepsilon_{i}(\nu)$ the complex dielectric function of the material in the $i$ th layer of the sample. The $x$-component of the electric field can be obtained from Maxwell's equation

$$
E_{x}^{(i)}(x, y)=\frac{1}{j \omega \varepsilon_{i}(\nu)} \frac{\partial\left[H_{z}^{(i)}(x, y)\right]}{\partial y} .
$$

According to the continuity of $H_{z}^{(i)}(x, y)$ and $E_{x}^{(i)}(x, y)$ at the boundary $y=D_{i}(i=1,2, \ldots, L)$, we can get the following iterative formula:

$$
\begin{aligned}
A_{i}= & {\left[A_{i+1}\left(1+\tau_{i}\right) \exp \left(-j \beta_{i+1} D_{i}\right)\right.} \\
& \left.+B_{i+1}\left(1-\tau_{i}\right) \exp \left(j \beta_{i+1} D_{i}\right)\right] \exp \left(j \beta_{i} D_{i}\right) / 2 \\
B_{i}= & {\left[A_{i+1}\left(1-\tau_{i}\right) \exp \left(-j \beta_{i+1} D_{i}\right)\right.} \\
& \left.+B_{i+1}\left(1+\tau_{i}\right) \exp \left(j \beta_{i+1} D_{i}\right)\right] \exp \left(-j \beta_{i} D_{i}\right) / 2
\end{aligned}
$$


where $\tau_{i}=\varepsilon_{i} \beta_{i+1} / \varepsilon_{i+1} \beta_{i}$. Given arbitrarily $A_{L}=1, B_{L}=0$, $A_{1}$ and $B_{1}$ were obtained by iterating the aforementioned equations. The reflection coefficient was then calculated as $B_{1} / A_{1}$ and the transmission as $A_{L} / A_{1}$.

In the numerical calculation, we used a Lorentz model [16] with $M$-oscillators to express the complex dielectric function of the material in the $i$ th layer of the sample

$$
\begin{aligned}
\varepsilon_{i}(\nu) \equiv & n_{i}(\nu)+j \frac{\alpha(\nu) c}{4 \pi \nu} \\
= & \sum_{m=1}^{M} \frac{S_{m}^{(i)}\left(\nu_{m}^{(i)}\right)^{2}}{\left(\nu_{m}^{(i)}\right)^{2}-\nu^{2}-j \nu \Gamma_{m}^{(i)}} \\
& +\left[n_{0}^{(i)}-j\left(C_{1}^{(i)}+C_{2}^{(i)} \nu+C_{3}^{(i)} \nu^{2}+C_{4}^{(i)} \nu^{3}\right)\right]^{2}
\end{aligned}
$$

where $v_{n}^{(i)}, \Gamma_{n}^{(i)}$, and $S_{n}^{(i)}$ are the center frequency, the width, and the strength of the $m$ th oscillator $(m=1,2, \ldots M)$ for the $i$ th layer $(i=1,2, \ldots, L)$. The second item in the bracket is an empirical expression for the extinction coefficient (caused by the scattering, for example). As an example, Fig. 4 shows the simulated extinction coefficient and refractive index of a pharmaceutical active ingredient, which has two spectral features at 31 and $52 \mathrm{~cm}^{-1}(0.93$ and $1.56 \mathrm{THz})$. Note that the Lorentz oscillator will introduce spectral features in both the refractive index spectrum and the extinction coefficient spectrum. With (2), (4), and (5), one can simulate terahertz waveform (reflection and transmission) from a sample with any number of coating layers. In order to facilitate discussion, we provide the analytical expression of a terahertz waveform reflected from a sample with a single coating layer, under normal incident condition

$$
\begin{aligned}
R(v)= & \frac{B_{1}(v)}{A_{1}(v)} \equiv r_{1}(v) \\
& +\frac{\left[1-r_{1}(v)\right]\left[1+r_{1}(v)\right] r_{2}(v) \exp \left(-j 2 \beta_{2}(v) D\right)}{1+r_{1}(v) r_{2}(v) \exp \left(-j 2 \beta_{2}(v) D\right)}
\end{aligned}
$$

where $D$ is the coating thickness, and

$$
\begin{aligned}
& r_{1}(v)=\frac{1-\tau_{1}(v)}{1+\tau_{1}(v)}=\frac{\sqrt{\varepsilon_{2}(v)}-\sqrt{\varepsilon_{1}(v)}}{\sqrt{\varepsilon_{2}(v)}+\sqrt{\varepsilon_{1}(v)}} \\
& r_{2}(v)=\frac{1-\tau_{2}(v)}{1+\tau_{2}(v)}=\frac{\sqrt{\varepsilon_{3}(v)}-\sqrt{\varepsilon_{2}(v)}}{\sqrt{\varepsilon_{3}(v)}+\sqrt{\varepsilon_{2}(v)}} .
\end{aligned}
$$

In order to understand the physical meaning of (6), we use the Taylor expansion to rewrite (6) [for readability, we omit $(\nu)$ in the expression]

$$
\begin{aligned}
R=r_{1} & +\left(1-r_{1}^{2}\right) r_{2}\left\{\exp \left(-j 2 \beta_{2} D\right)-r_{1} r_{2} \exp \left(-j 4 \beta_{2} D\right)\right. \\
& \left.+r_{1}^{2} r_{2}^{2} \exp \left(-j 6 \beta_{2} D\right)-\cdots\right\} .
\end{aligned}
$$

It is clear now that the first item $\left(r_{1}\right)$ is the terahertz reflection from the sample surface while the second item represents terahertz multiple reflections within the coating layers, with the items in the bracket corresponding to the "fundamental" reflection (the first item in the bracket) and "parasitic" reflections (the

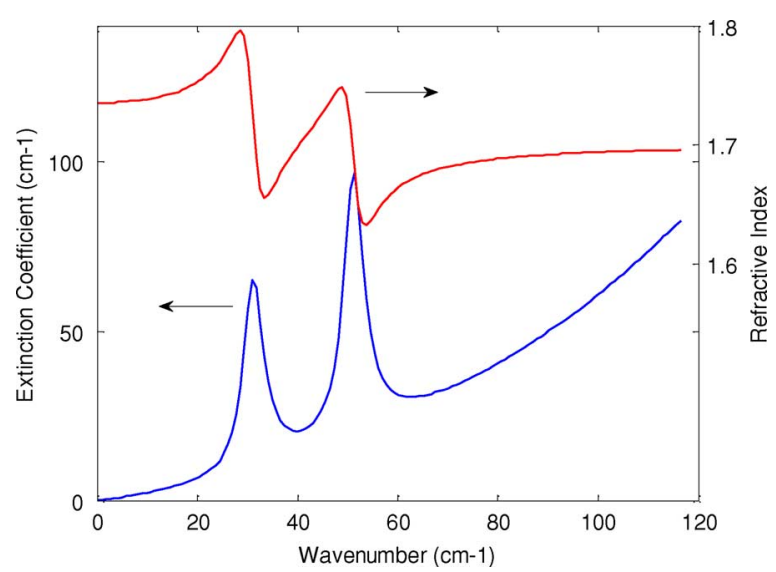

Fig. 4. Simulated extinction coefficient and refractive index using a Lorentz model. In the simulation, the center frequency values of the two oscillators are 31 and $52 \mathrm{~cm}^{-1}(0.93$ and $1.56 \mathrm{THz})$, respectively; the width of both the oscillators is $5 \mathrm{~cm}^{-1}(0.15 \mathrm{THz})$; the strengths of the two oscillators are 80 and 40, respectively; and $C_{1}=C_{2}=C_{3}=0.2$, and $C_{4}=0$.

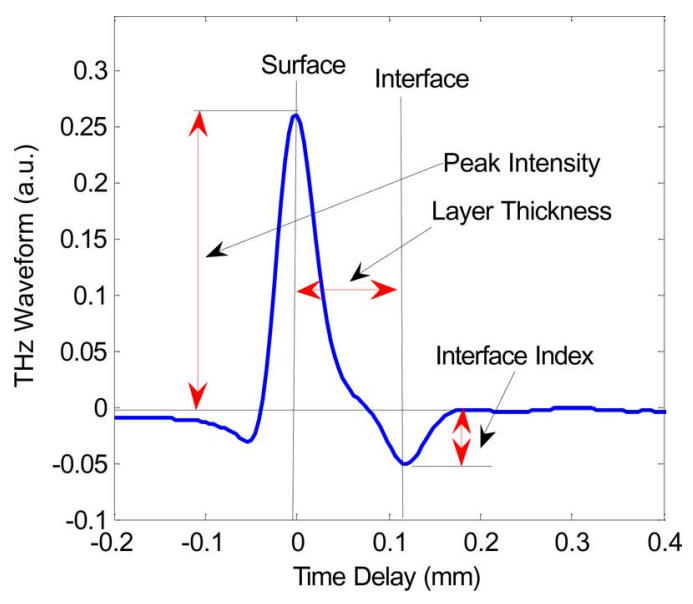

Fig. 5. Typical terahertz waveform measured for a coated tablet. Arrows indicate how the peak intensity, layer thickness, and interface index were determined.

following items in the bracket). Note that the terahertz waveform can be obtained by Fourier transforming (8), and the multiple reflections will be separated in time domain owing to their phase difference $\left(\exp \left(-j 2 \beta_{2} D\right)\right)$.

\section{B. Terahertz Waveform and Coating Analysis}

Terahertz pulses incident on a tablet surface penetrate through different coating layers. At each interface or abrupt change in refractive index, a portion of the terahertz pulse is reflected back to the detector. To illustrate the principle of coating analysis, Fig. 5 shows an example terahertz time-domain waveform recorded at the center of a coated tablet (single-point measurement). The maximum (at $0.0 \mathrm{~mm}$ ) is due to reflection of the terahertz pulse at the tablet surface (air/coating interface). The minimum (at 0.11 $\mathrm{mm}$ ) arises from terahertz reflection from the coating/core interface. Three parameters can be obtained: 1) Peak Amplitude: The amplitude of the main peak, which is an indicator of the refractive index of the tablet surface; 2) Coating Thickness: Peak position difference between the surface reflection and the interface 


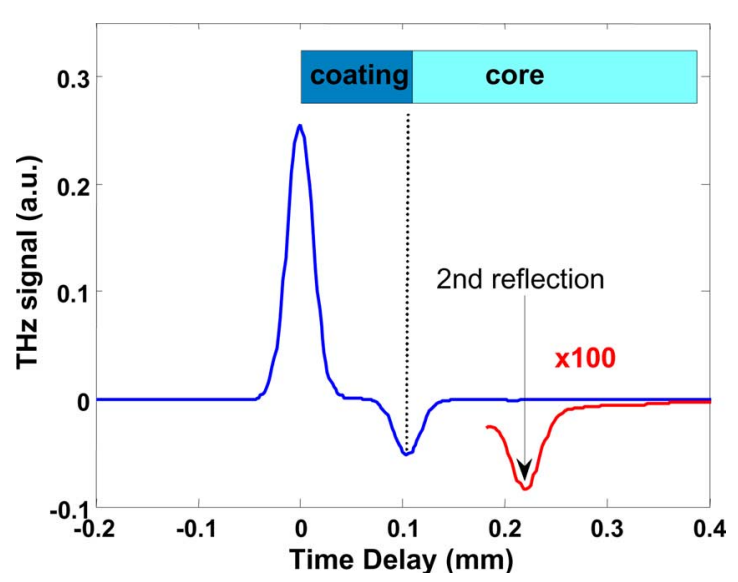

Fig. 6. Terahertz waveform calculated for a coated tablet. The refractive index values are 1.68 and 1.5 for coating and core, respectively. The coating thickness is $66 \mu \mathrm{m}$. Note that the strength of the "parasitic" reflection (at $0.22 \mathrm{~mm}$ ) is much smaller than that of the "fundamental" reflection (at $0.11 \mathrm{~mm}$ ).

reflection, divided by the refractive index value of tablet coating (note that the terahertz path length is twice that of the layer thickness); 3) Interface Index: Peak height ratio of the interface reflection and the surface reflection (in percentage), which gives a measure of the strength of reflection from the buried surface and indicates the change in physical or chemical composition at the interface.

In order to determine the absolute layer thickness, one needs to know precisely the value of the refractive index of each layer. This can be accomplished by establishing a terahertz spectrum library of all pharmaceutical ingredients and excipients of interest. Alternatively, the refractive index values can be calculated using (8). For example, the refractive index of the first coating layer $\left(n_{2}\right)$ can be estimated from the following equation: $R_{0}=\left(n_{2}-1\right) /\left(n_{2}+1\right)$, where $R_{0}$ is the surface reflection peak. The refractive index of the inner layer $\left(n_{3}\right)$ can also be estimated in a similar manner using the following equation: $R_{1}=4 n_{2} /\left(n_{2}+1\right)^{2}\left(n_{3}-n_{2}\right) /\left(n_{3}+n_{2}\right)$, where $n_{2}$ has been predetermined and we assume that the terahertz absorption of the thin coating layer can be neglected. For the terahertz waveform shown in Fig. 5, the refractive index values of the coating and core were estimated to be 1.68 and 1.5 , respectively, and the layer thickness was determined to be $66 \mu \mathrm{m}$. With the knowledge of the coating layer thickness and the refractive indices of the coating and core, the terahertz waveform can be calculated using the electromagnetic theory discussed in Section III-A. Fig. 6 shows such a calculated terahertz waveform (coating thickness $66 \mu \mathrm{m}$, refractive index values are 1.68 and 1.5 for coating and core, respectively). As evident from Figs. 5 and 6 , the calculated peak height and peak position agree well with the measured one.

In addition, there are multiple backreflections within the coating (as indicated by an arrow in Fig. 6). However, despite the over $10 \%$ change in the refractive index value between the coating and the core, the strength of the first "parasitic" reflection (at $0.22 \mathrm{~mm}$ ) is less than two hundredths of that of the "fundamental" reflection (at $0.11 \mathrm{~mm}$ ). Therefore, for most pharmaceutical samples, the contribution of multiple backreflections is

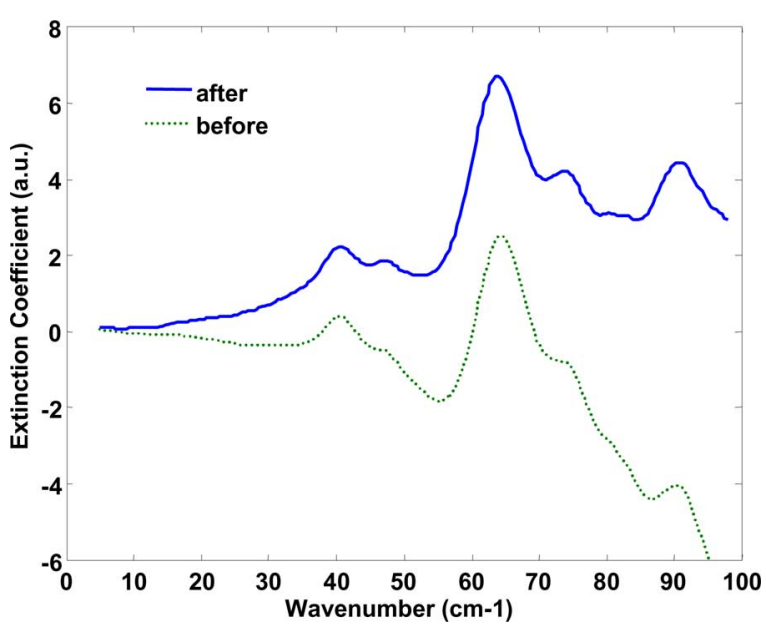

Fig. 7. Absorption spectrum calculated from the TPI measurement before (top curve) and after (lower curve) phase compensation. A constant phase shift of $\Delta x=7$ was used in the calculation.

not significant. Nevertheless, "parasitic" reflection may become important for products with lamination problems (presence of air gap between coating and core), where the change in refractive index at the interface is large. On the other hand, a large "fundamental" reflection itself usually indicates the failure of the pharmaceutical product under investigation.

\section{Terahertz Spectra and Chemical Mapping}

In TPI measurement, the electric field of terahertz radiation was recorded as a function of time. Terahertz spectral information was obtained by Fourier transforming time-domain terahertz waveform into frequency domain. The absorption coefficient spectrum, $\alpha(\nu)$, and the refractive index spectrum, $n(\nu)$, were then calculated as follows:

$$
\sqrt{\varepsilon} \equiv n(\nu)+j \frac{\alpha(\nu) c}{4 \pi \nu}=\frac{1-R(\nu)}{1+R(\nu)}
$$

where $R(\nu) \equiv E_{s}(\nu) / E_{M}(\nu)$ is the frequency-dependent reflection coefficient, and $E_{S}(\nu)$ and $E_{M}(\nu)$ are the Fourier transforms of the terahertz waveform reflected/measured for a sample and a reference mirror, respectively. It is worthwhile to note that in real measurement, the sample and the reference may not be in the exact same position. Consequently, the true reflectance will take the form as: $R^{\prime}(\nu)=R(\nu) e^{j k(\nu) 2 \Delta x}$. Here $\Delta x$ is the position difference between the reference and sample measurements. Clearly, this will introduce an additional imaginary component in the equation and, thus, give problems when calculating the absorption coefficient. One approach to extract the absorption coefficient of the sample is to introduce an additional phase shift $\left(e^{-j k(\nu) 2 \Delta x}\right)$ into the reflection coefficient. This compensates the phase shift caused by the imperfection of the measurement, such as the rough surface of the sample. As the first approximation, we currently used a constant $\Delta x$ value (at each pixel) to compensate for all frequency components. Fig. 7 shows, as an example, the extracted extinction coefficient from the measured terahertz waveform before and after the phase shift compensation. We note that the terahertz beam size is frequency 
dependent; thus, the terahertz component at different frequencies may experience different surface roughness. Nevertheless, using a constant $\Delta x$ value still provides satisfactory results for most samples studied.

Once the absorption and refractive index spectra are determined, various spectral matching techniques can be used to generate chemical maps. We used the cosine correlation mapping (CCM) for chemical mapping [17]. For this purpose, we define a pixel vector as $p$ (terahertz spectrum measured for the sample under investigation) and a target vector as $t$ (terahertz spectrum measured for the reference sample). The number of hyperspectral channels is $\mathbf{n}$ (the number of the data points of the spectral data). The similarity of the pixel and the target vector can be represented as

$$
\cos (\theta)=\sum_{i=1}^{n} t_{i} p_{i} / \sqrt{\sum_{i=1}^{n} t_{i}^{2}} \sqrt{\sum_{i=1}^{n} p_{i}^{2}}
$$

where $\theta$ is the angle between the pixel vector and the target vector, and the value of $\cos (\theta)$ is between 0 and 1 . The smaller angle means more similarity between the pixel and the target spectra. Note that both the absorption spectrum and the refractive index spectrum can be used for calculating the chemical map, and a combined use of both the absorption feature and the refractive index feature is a better way for chemical identification/mapping.

In the pharmaceutical industry, the extracted chemical maps are usually displayed as an RGB map. For example, the value of $\cos (\theta)$ obtained for chemical $\mathrm{A}$ is used to assign red color $[r, 0,0]$ of the RGB map, where $r$ is an integral and has a value of $255 \times \cos (\theta)$. The green color $[0, g, 0]$ and blue color $[0,0, b]$ are assigned in a similar manner. One of the advantages of the RGB map is that it can display three chemicals in a single map, thus allowing better visualization of chemical distributions of a sample.

\section{Terahertz Spectrogram and 3-D Chemical Mapping}

In the previous two sections, we discussed coating analysis using the time-domain terahertz waveform, and chemical mapping using the frequency-domain terahertz spectrum. The absorption features in the terahertz frequency range are mostly caused by intermolecular interaction. Therefore, the amorphous material has no sharp absorption features while the crystalline material does. The spectral absorption features in the frequency domain will lead to oscillating features in the time domain. Consequently, the measured time-domain terahertz signal will be a coupling mixture of both the structural and chemical features. Fig. 8 shows the simulated terahertz waveforms reflected from a three-layered sample (coating layer, drug layer, and core). When the drug is amorphous, the reflected terahertz waveform [curve (a)] is "clean" and only has two interface reflection features (coating/drug interface at $0.3 \mathrm{~mm}$, and drug/core interface at $0.5 \mathrm{~mm}$ ). However, for the crystalline drug, the terahertz signal [curve (b)] has much more complicated waveform shape including oscillation-like features caused by resonance absorption (in the simulation, we used the refractive index and extinction co-

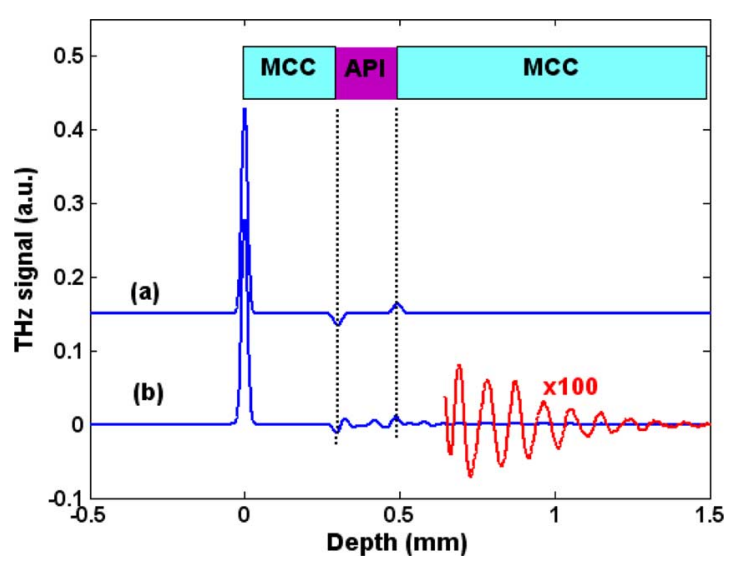

Fig. 8. Simulated terahertz waveform reflected from a layered sample. (a) The API is amorphous and has no sharp spectral feature. (b) The API is crystalline and has sharp absorption signature. MCC: Microcrystalline cellulose, which is a common excipient; API: Active pharmaceutical ingredient, which is theophylline, in this specific case.

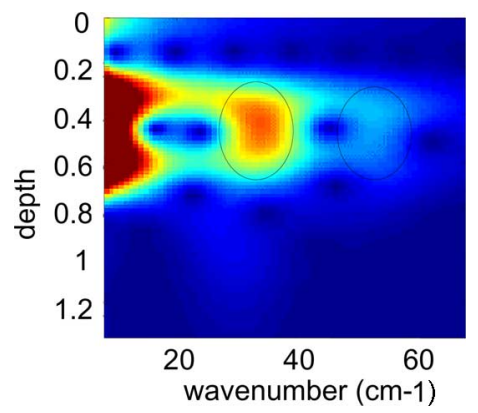

Fig. 9. Terahertz spectrogram calculated on the terahertz waveform, shown in Fig. 8, using the windowed Fourier transform $(\Delta t=0.2$.)

efficient spectra data, shown in Fig. 4 for the crystalline drug).

One method to separate the structural and chemical information is to calculate the spectrogram of the terahertz waveform using the windowed Fourier transform [18]

$$
\begin{aligned}
R(\nu, \tau)= & \int_{-\infty}^{\infty} E_{S}^{\mathrm{THz}}(t) \exp \left[-(t-\tau)^{2} /(\Delta t)^{2}\right] \\
& \times \exp (j 2 \pi \nu t) d t / \int_{-\infty}^{\infty} E_{R}^{\mathrm{THz}}(t) \exp (j 2 \pi \nu t) d t
\end{aligned}
$$

where $E_{S}^{\mathrm{THz}}$ and $E_{R}^{\mathrm{THz}}$ are the terahertz signals measured for a sample and a reference, respectively. Fig. 9 shows the calculated spectrogram map of the terahertz waveform shown in curve (b) of Fig. 8 . The $x$-axis is frequency (in $\mathrm{cm}^{-1}$ ) and the $y$-axis is depth (in $\mathrm{mm}$ ). With the prior knowledge of the absorption features of the theophylline $\left(31\right.$ and $52 \mathrm{~cm}^{-1}$, or 0.93 and $1.56 \mathrm{THz}$ ), we can conclude that there is presence of drug at $0.2-0.5 \mathrm{~mm}$. In this calculation, we used a Gaussian window of width $0.2 \mathrm{~mm}$, although other types of window functions can also be used. Note that a Gaussian window with a larger width will provide better spectral resolution but compromise the depth resolution. More research into advanced spectral-time analysis 
(a)
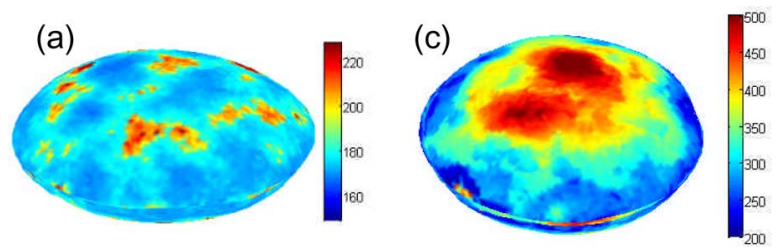

(b)

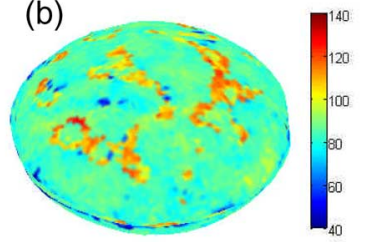

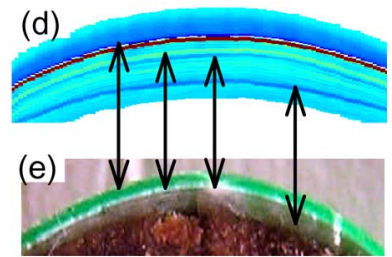

Fig. 10. (a)-(c) Coating thickness maps of three-layered structures of an M\&M chocolate. (d) and (e) The TPI map and the photograph of a cross-section, respectively. Solid arrows are guides to the eye.

techniques is needed in order to realize the full potential of the 3-D chemical mapping capability of the TPI.

\section{RESULTS AND DISCUSSION}

\section{A. Coating Thickness Map}

Terahertz 3-D maps comprising approximately 4000 point measurements were recorded for an M\&M chocolate. Each point measurement gives a terahertz waveform of 512 data points. The recorded TPI cube was analyzed to generate TPI maps, such as the coating thickness map for each observed layer, as shown in Fig. 10(a)-(c). These coating thickness maps show the uniformity of the coating layer over the tablet and can be used to identify inhomogeneities or defects in individual tablet coating of pharmaceutical products. Fig. 10(d) shows the TPI crosssection map. For comparison, the photograph of a cut M\&M is shown in Fig. 10(e). We can clearly see three distinct layered structures, i.e., the green outer layer, the white intermediate layer, and the grey inner layer, and the chocolate core. The mean and standard deviation of the layer thickness were determined to be $183 \pm 11 \mu \mathrm{m}, 87 \pm 10 \mu \mathrm{m}$, and $338 \pm 72 \mu \mathrm{m}$ for the green outer layer, the white intermediate layer, and the grey inner layer, respectively. The refractive indices estimated were $1.8,1.85$, and 1.7 for the green outer layer, the white intermediate layer, and the grey inner layer, respectively. Note that the standard deviation value here is determined by the layer thickness variation of the sample itself, not by the TPI measurement.

In this paper, we mainly use the time-domain terahertz waveform for determining layer thickness. This method is fast, robust, and intuitive (peaks in terahertz waveform correspond to the terahertz reflections from the surface/interfaces). We noted that material properties including layer thickness can also be extracted by analyzing the frequency-domain terahertz spectrum [19]-[21], and we will explore this further in future.

\section{B. 3-D Structural Mapping}

In a TPI measurement, the terahertz waveform for each pixel was recorded as a function of optical time delay. This provides

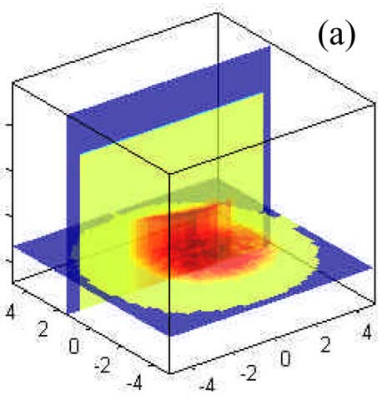

(a)

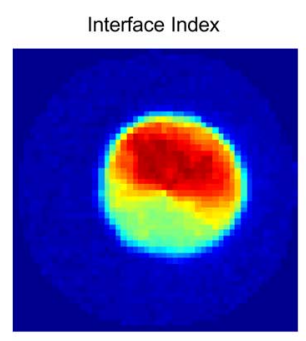

(c)
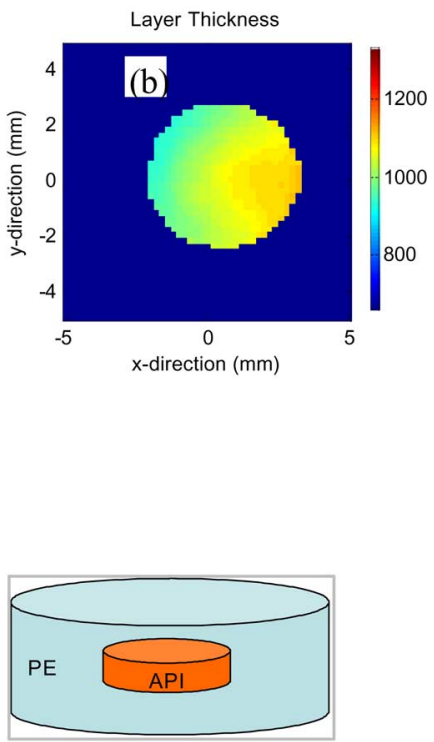

(d)
Fig. 11. (a) $z$ - and $x$-cross-section view of the 3-D TPI data set. (b) Layer thickness map. (c) Interface index map of the PE/API interface. (d) Schematic diagram of the sample under investigation. PE and API are polyethylene and active pharmaceutical ingredient (though, it is lactose, in this particular case), respectively.

a 3-D data set, where two axes describe vertical and horizontal spatial dimensions, and the third axis represents the time-delay (depth) dimension. Therefore, the TPI is a true 3-D imaging tool and the structure map of a sample can be fully reconstructed by analyzing the measured TPI data set. To illustrate the 3-D structural mapping capability, a pellet sample comprising a piece of lactose embedded in polyethylene (PE) was measured. Fig. 11(a) shows a 3-D view of the reconstructed PE pellet sample, where the buried lactose in the pellet is revealed. The reconstructed 3-D structural maps are in good agreement with those obtained using the X-ray computed topography (CT) technique [22]. Both the X-ray CT and the TPI are nondestructive and provide 3-D structural information (density and/or refractive index variation) of tablets. However, the TPI directly measures the electric field of terahertz radiation as a function of time, which is the square-root of the terahertz radiation intensity, thus providing intrinsic higher sensitivity in detecting small refractive index changes inside a sample. Furthermore, the TPI also provides additional chemical information (terahertz spectral signatures) of pharmaceutical tablets, and this will be discussed in the next section.

In addition, the quality of the curved interface between PE and lactose, which cannot be analyzed using the $\mathrm{X}$-ray $\mathrm{CT}$, was assayed as the interface index map using the TPI, as shown in Fig. 11(c). As mentioned earlier, the interface index map is a measure of the strength of reflection from the buried surface and, thus, gives an indication of the change in physical and/or chemical properties at the interface. In this particular case, the red color (stronger terahertz reflection) indicates areas with loose joint (presence of air bubbles in the compacted PE), that is, capping occurring at the interface. Therefore, the interface index 


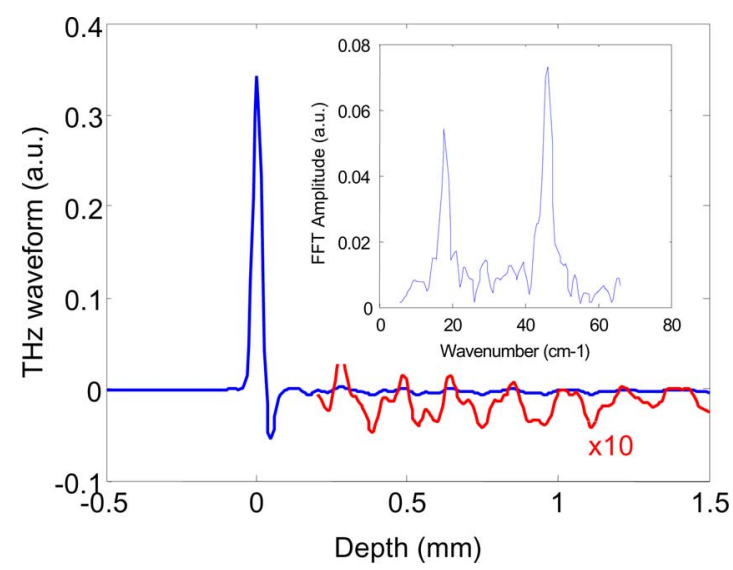

Fig. 12. Typical terahertz waveform measured for a coated tablet. The ripplelike oscillations immediately after the first negative peak are caused by resonant terahertz absorptions. The inset shows the Fourier transform amplitude of the terahertz waveform in a window of $0.1-1.5 \mathrm{~mm}$.

map is a better indicator in identifying structural defects such as the delamination, capping, or cracking inside a pharmaceutical matrix.

\section{Structural and Spectral Features}

In the previous two sections, we demonstrated that the TPI is a powerful tool for characterizing the inner structures of the pharmaceutical samples. On the other hand, many pharmaceutical ingredients and excipients have resonance absorption features in the terahertz frequency range. The fingerprint features in the frequency domain will lead to oscillations in the time-domain terahertz signal, thus interfering in the coating analysis. Fig. 12 shows a terahertz waveform measured for a coated tablet. The first positive peak (peak amplitude is about 0.335 ) is terahertz reflection from the sample surface, while the first negative peak at $48 \mu \mathrm{m}$ (peak amplitude is about 0.053 ) is the reflection peak from the coating/core interface. Using (7) and (8), the refractive index of the coating and core were estimated to be approximately 2.0 and 1.77 , respectively. In addition, there are ripple-like oscillations immediately after the negative peak. These features could be either spectral features or structural features (various coating layers or the same coating layer with various densities). One method to distinguish the structural feature from the spectral feature is to calculate windowed Fourier transform (as shown in the inset of Fig. 12). The Fourier transform of the terahertz waveform in the window of $0.1-1.5 \mathrm{~mm}$ shows two distinct features at 18 and $46 \mathrm{~cm}^{-1}(0.54$ and $1.38 \mathrm{THz})$. From the previous analysis, we can identify the sample as a $\beta$-lactose sample covered with a $46-\mu \mathrm{m}$ thick coating layer.

\section{Chemical Mapping for Identifying Polymorphs}

One of the most predominate features of the terahertz technique is its capability for polymorphic discrimination. In the terahertz frequency range, the absorption features come from the intermolecular interactions, rather than intramolecular interactions. Therefore, the terahertz spectral analysis provides a direct method for characterizing polymorphs of chemicals [23], [24].
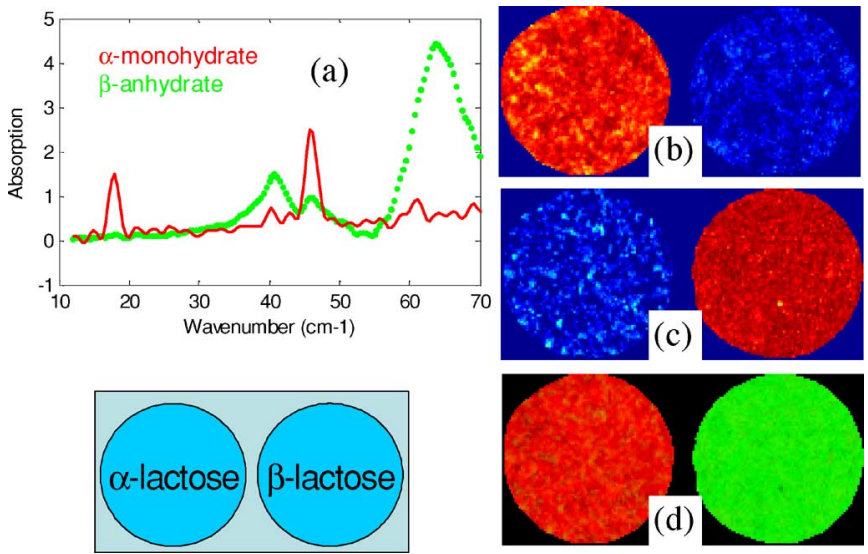

Fig. 13. (a) Typical terahertz spectra. (b) and (c) Chemical maps of $\alpha$ monohydrate and $\beta$-anhydrate lactose, respectively. (d) The RGB map of the sample. The inset of (a) shows the schematic diagram of the imaged sample.

Here, we use the $\alpha$-monohydrate and $\beta$-anhydrate lactose to illustrate the chemical mapping capability of the TPI. Fig. 13(b) and (c) shows the chemical map reconstructed using the terahertz spectrum of the $\alpha$-monohydrate and $\beta$-anhydrate lactose, with Fig. 13(d) showing the RGB map of the sample. Fig. 13(a) shows the typical terahertz spectrum obtained at the $\alpha$-lactose and $\beta$-lactose regions, respectively. It is clear that the $\alpha$-monohydrate and $\beta$-anhydrate lactose can be easily mapped and identified using the TPI. This chemical specificity of the TPI technology is very useful for characterizing the pharmaceutical products. For example, most pharmaceutical tablets are formed by compressing powder mixtures of the API and the excipients. The API might undergo polymorph transition during compression process or storage, and, currently, there is no technique capable of detecting these changes nondestructively. As demonstrated here, the TPI technology has the full potential to detect/map such transitions nondestructively.

\section{E. 3-D Chemical Mapping}

As demonstrated earlier, the TPI has the necessary chemical specificity and the penetration capability desired for 3-D chemical mapping. Recently, with the significant technological advances in terahertz source and detector, chemical mapping either at sample surface or through a sample has been reported [25]-[27]. However, the 3-D chemical mapping, one of the most desirable and promising applications of the TPI, is still missing from scientific literature. One of the key issues behind the deceptive simplicity of the terahertz 3-D chemical mapping principle is the spatial-temporal-spectral coupling of terahertz radiations. As shown in Fig. 8, the simulated terahertz waveform reflected from a layered sample is actually a coupling mixture of the structural contribution (layer interfaces at 0.3 and $0.5 \mathrm{~mm}$ ) and the chemical contribution [oscillating features of curve (b) are caused by resonant absorption of crystalline API]. The high-frequency oscillation, which is the key for chemical mapping, has a very small amplitude $(\sim 0.1 \%$ of the input terahertz radiation strength). Worse still, most pharmaceutical tablets are formed by compressing a complex mixture 

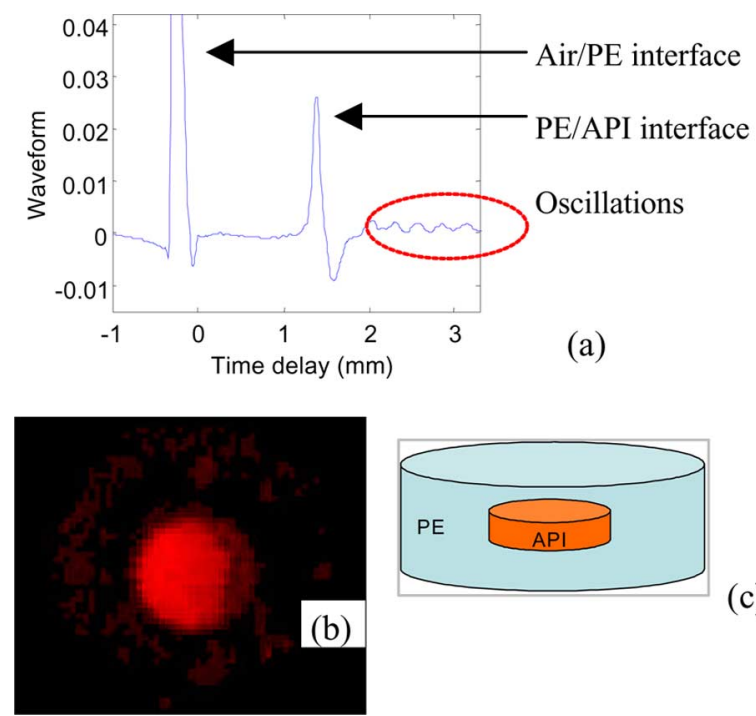

(b)

Fig. 14. 3-D chemical mapping capable of not only mapping the internal structures of a sample, but also identifying their chemical identities. (a) Terahertz waveform contains both the structural information (reflection peaks at interfaces) and the spectral information (oscillations in time domain correspond to spectral signature in frequency domain). (b) The RGB map showing the chemical distribution of the API inside the sample. (c) Schematic diagram of the sample under investigation. PE and API are polyethylene and active pharmaceutical ingredient (though, it is lactose, in this particular case).

of chemical powder; thus, they could have many particles (density/chemical variations) leading to strong scattering. This will not only attenuate the already very weak oscillating features but also complicate the data analysis due to the additional features in the terahertz signal introduced by the reflection/scattering events. Therefore, a full 3-D chemical mapping functionality requires advanced technology capable of detecting, separating, and recording the extremely weak signatures from the reflected and scattered terahertz pulses. Nevertheless, under favorable conditions, the 3-D chemical maps can be obtained using the TPI. Fig. 14 shows such an example where the API inside a compressed pellet is successfully mapped and, subsequently, identifies using its spectral signatures. The windowed Fourier transform, discussed in Section III-D, was used to calculate the terahertz spectrogram, which was then used to generate the terahertz map.

\section{F. Lateral and Depth Resolution}

Spatial resolution is one of the key performance parameters of an imaging system. As discussed earlier, the TPI provides 3-D structural maps of a sample and, therefore, the spatial resolution should include both the lateral spatial resolution and the axial (depth direction) spatial resolution. Here, we used traceably calibrated samples purchased from the NIST (Gaithersburg, USA) to evaluate the depth resolution; while a star-pattern sample (nickel on gallium arsenide wafer) fabricated at the Cavendish Laboratory (Cambridge, U.K.) was used to characterize the lateral spatial resolution. As shown in Fig. 15, the measured layer thickness values agree well with the layer thickness specified by the NIST (A refractive index of 1.57 was obtained from the terahertz transmission spectroscopy measurement and was, sub-

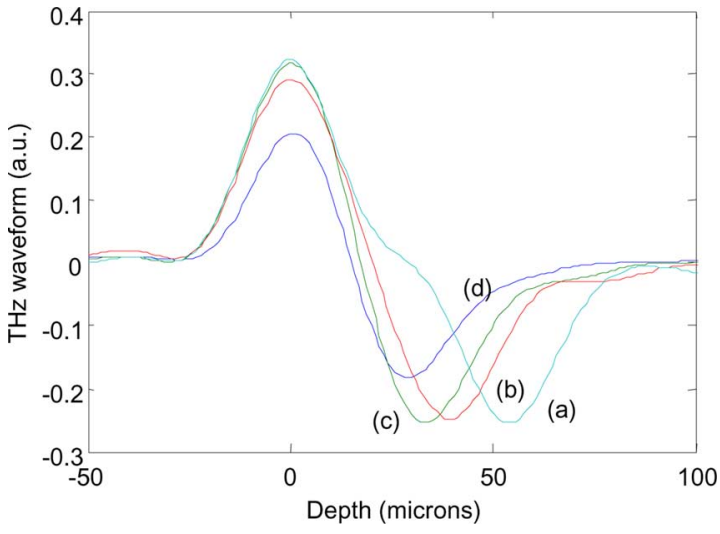

Fig. 15. Measured terahertz waveform for the stand-alone standard NIST samples with a calibrated layer thickness of (a) $26 \mu \mathrm{m}$, (b) $32 \mu \mathrm{m}$, (c) $36 \mu \mathrm{m}$, and (d) $51 \mu \mathrm{m}$. A refractive index of 1.57 was determined from terahertz transmission measurements and was subsequently used for calculating the layer thickness. The layer thickness determined from the TPI measurement agree well with the numbers provided by the NIST.
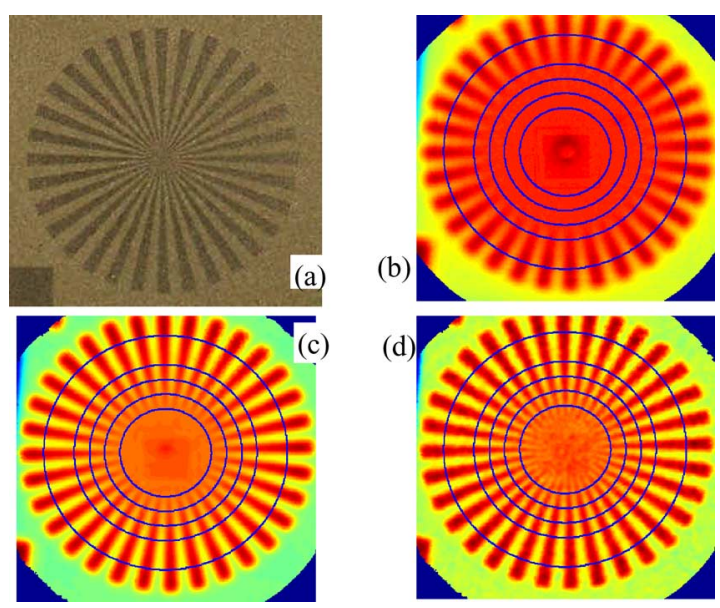

Fig. 16. (a) Photograph of a calibration sample. (b)-(d) TPI maps at a wavenumber of 30,60 , and $90 \mathrm{~cm}^{-1}(0.9,1.8$, and $2.7 \mathrm{THz})$, respectively. The sample used is a nickel pattern on a GaAs wafer and the blue circles in the TPI maps correspond to a periodical spacing of 400, 300, 250, 200, and $150 \mu \mathrm{m}$, respectively.

sequently, used for calculating the layer thickness here.). The limit of detection of the layer thickness was determined to be approximately $30 \mu \mathrm{m}$, a fraction of the shortest wavelength of the terahertz pulse. Fig. 16 shows the TPI maps of the starpattern sample at various terahertz frequencies. As expected, the lateral spatial resolution is frequency dependent and a spatial resolution of $150 \mu \mathrm{m}$ can be achieved at $90 \mathrm{~cm}^{-1}$ (frequency $2.7 \mathrm{THz}$, wavelength $111 \mu \mathrm{m}$ ). Note that the lateral resolution is finally determined by the confinement of terahertz pulses in space, and, therefore, will be frequency dependent, and be ultimately diffraction limited by the wavelength (no better than the half of the wavelength). The axial spatial resolution is determined by the confinement of the terahertz pulse in time (pulse width) and, thus, could be smaller than the shortest wavelength of a terahertz pulse. As demonstrated here, the axial spatial resolution is approximately five times better than the lateral spatial resolution. We note that the axial resolution can be further improved by using an interferometric imaging approach, where 
the layer thickness down to $10 \mu \mathrm{m}$ can be resolved [28]. On the other hand, the 3-D structural information can also be obtained using the terahertz computed tomography [29], but the spatial resolution will be Rayleigh limited.

\section{CONCLUSION}

In this paper, we have demonstrated the capability of the TPI for nondestructive evaluation of the 3-D structures of pharmaceutical solid dosage forms. Critical quality issues of pharmaceutical products, such as coating uniformity and integrity, and tablet uniformity in the 3-D and polymorphs, were obtained using the TPI. Furthermore, we demonstrated that the TPI has the necessary penetration depth and chemical specifiticity to afford 3-D chemical mapping, but technological advancements on the data processing will be required to fully utilize the technology.

\section{REFERENCES}

[1] M. J. Kottk and E. M. Rudnic, "Tablet dosage forms," in Modern Pharmaceutics, 4th ed., G. S. Banker and C. T. Rhodes, Eds. New York: Marcel Dekker, 2002, pp. 287-334.

[2] G. M. Jantzen and J. R. Robinson, "Sustained and controlled-release drug delivery systems," in Modern Pharmaceutics, 4th ed., G. S. Banker and C. T. Rhodes, Eds. New York: Marcel Dekker, 2002, pp. 501-528.

[3] G. Reich, "Near-infrared spectroscopy and imaging: Basic principles and pharmaceutical applications," Adv. Drug. Deliv. Rev., vol. 57, pp. 1109$1143,2005$.

[4] F. Clarke, "Extracting process-related information from pharmaceutical dosage forms using near infrared microscopy," Vib. Spectrosc., vol. 34, pp. 25-35, 2004.

[5] I. C. Sinka, S. F. Burch, J. H. Tweed, and J. C. Cunningham, "Measurement of density variations in tablets using X-ray computed tomography," Int. J. Pharm., vol. 271, pp. 215-224, 2004.

[6] V. Busignies, B. Leclerc, P. Porion, P. Evesque, G. Couarraze, and P. Tchoreloff, "Quantitative measurements of localized density variations in cylindrical tablets using X-ray microtomography," Eur. J. Pharm. Biopharm., vol. 64, pp. 38-50, 2006.

[7] P. F. Taday, I. V. Bradley, D. D. Arnone, and M. Pepper, "Using terahertz pulse spectroscopy to study the crystalline structure of a drug: A case study of the polymorphs of ranitidine hydrochloride," J. Pharm. Sci., vol. 92 , pp. 831-838, 2003.

[8] X. C. Zhang, "Terahertz wave imaging: Horizons and hurdles," Phys. Med. Biol., vol. 47, pp. 3667-3677, 2002.

[9] A. J. Fitzgerald, B. E. Cole, and P. F. Taday, "Nondestructive analysis of tablet coating thicknesses using terahertz pulsed imaging," J. Pharm. Sci., vol. 94, pp. 177-183, 2005.

[10] J. A. Zeitler, Y. C. Shen, C. Baker, P. F. Taday, M. Pepper, and T. Rades, "Analysis of coating structures and interfaces in solid oral dosage forms by three dimensional terahertz pulsed imaging," J. Pharm. Sci., vol. 96, pp. 330-340, 2007.

[11] J. A. Zeitler, P. F. Taday, D. A. Newnham, M. Pepper, K. C. Gordon, and T. Rades, "Terahertz pulsed spectroscopy and imaging in the pharmaceutical setting-a review," J. Pharm. Pharmacol., vol. 59, pp. 209-223, 2007.

[12] L. Ho, R. Müller, M. Römer, K. C. Gordon, J. Heinämäki, P. Kleinebudde, M. Pepper, T. Rades, Y. C. Shen, C. J. Strachan, P. F. Taday, and J. A. Zeitler, "Analysis of sustained-release tablet film coats using terahertz pulsed imaging," J. Control Release, vol. 119, pp. 253-261, 2007.

[13] P. R. Smith, D. H. Auston, and M. C. Nuss, "Subpicosecond photoconducting dipole antennas," IEEE J. Quantum Electron., vol. 24, no. 2, pp. 255-260, Feb. 1988.

[14] Y. C. Shen, P. C. Upadhya, H. E. Beere, E. H. Linfield, A. G. Davies, I. S. Gregory, C. Baker, W. R. Tribe, and M. J. Evans, "Generation and detection of ultrabroadband terahertz radiation with photoconductive emitter and receiver antennas," Appl. Phys. Lett., vol. 85, pp. 164-166, 2004.

[15] Y. C. Shen, S. Y. Zhang, and Y. S. Jiang, "Angular resonance absorption spectra of Langmuir-Blodgett films studied by the photoacoustic technique," Thin Solid Films, vol. 248, pp. 36-40, 1994.
[16] W. T. Silfvast, Laser Fundamentals, 2nd ed. Cambridge, U.K.: Cambridge Univ. Press, 2004, pp. 9-39.

[17] J. Schwarz and K. Staenz, "Adaptive threshold for spectral matching of hyperspectral data," Can. J. Remote Sens., vol. 27, pp. 216-224, 2001.

[18] Y. C. Shen, P. C. Upadhya, E. H. Linfield, and A. G. Davies, "Observation of far-infrared emission from excited cytosine molecules," Appl. Phys. Lett., vol. 87, p. 011105, 2005.

[19] L. Duvillaret, F. Garet, and J.-L. Coutaz, "Highly precise determination of optical constants and sample thickness in terahertz time-domain spectroscopy," Appl. Opt., vol. 38, pp. 409-415, 1999.

[20] T. Dorney, R. Baraniuk, and D. Mittleman, "Material parameter estimation with terahertz time-domain spectroscopy," J. Opt. Soc. Am. A, Opt. Image Sci., vol. 18, pp. 1562-1571, 2001.

[21] I. Pupeza, R. Wilk, and M. Koch, "Highly accurate optical material parameter determination with $\mathrm{THz}$ time domain spectroscopy," Opt. Express, vol. 15, pp. 4335-4350, 2007.

[22] Y. C. Shen and P. F. Taday. "Application of THz pulsed imaging for 3D non-destructive inspection of pharmaceutical tablet uniformity,"presented at IRMMW-THz-2007, Cardiff, U.K., Sep. 2-8

[23] P. F. Taday, I. V. Bradley, D. D. Arnone, and M. Pepper, "Using terahertz pulse spectroscopy to study the crystalline structure of a drug: A case study of the polymorphs of ranitidine hydrochloride" J. Pharm. Sci., vol. 92, pp. 831-838, 2003.

[24] J. A. Zeitler, D. A. Newnham, P. F. Taday, T. L. Threlfall, R. W. Lancaster, R. W. Berg, C. J. Strachan, M. Pepper, K. C. Gordon, and T. Rades, "Characterization of temperature-induced phase transitions in five polymorphic forms of sulfathiazole by terahertz pulsed spectroscopy and differential scanning calorimetry," J. Pharm. Sci., vol. 95, pp. 2486-2498, 2006.

[25] Y. Watanabe, K. Kawase, T. Ikari, H. Ito, Y. Ishikawa, and H. Minamide, "Component spatial pattern analysis of chemicals using terahertz spectroscopic imaging," Appl. Phys. Lett., vol. 83, pp. 800-802, 2003.

[26] Y. C. Shen, T. Lo, P. F. Taday, B. E. Cole, W. R. Tribe, and M. C. Kemp, "Detection and identification of explosives using terahertz pulsed spectroscopic imaging," Appl. Phys. Lett., vol. 86, p. 241116, 2005.

[27] H. Zhong, A. Redo-Sanchez, and X. C. Zhang, "Identification and classification of chemicals using terahertz reflective spectroscopic focal-plane imaging system," Opt. Express, vol. 14, pp. 9130-9141, 2006.

[28] J. L. Johnson, T. D. Dorney, and D. M. Mittleman, "Interferometric imaging with terahertz pulses," IEEE J. Sel. Topics Quantum Electron., vol. 7, no. 4, pp. 592-599, Jul.-Aug. 2001.

[29] B. Ferguson, S. H. Wang, D. Gray, D. Abbot, and X. C. Zhang, "T-ray computed tomography," Opt. Lett., vol. 27, pp. 1312-1314, 2002.

Yao-Chun Shen received the B.Sc. and M.Sc. degrees in electronics, and the Ph.D. degree in applied physics, all from Nanjing University, Nanjing, China.

He had a 3-year stint as a Postdoctoral Researcher at the Cavendish Laboratory, University of Cambridge, Cambridge, U.K., where he was engaged in research on the development of terahertz spectroscopy and imaging. Thereafter, he had a 3-year stint as a Senior Scientist with TeraView Ltd., Cambridge, where he was engaged in the exploitation of terahertz pulsed imaging applications. Since June 2007, he is a Senior Lecturer in the Department of Electrical Engineering and Electronics, University of Liverpool, Liverpool, U.K. His current research interests include terahertz pulsed spectroscopy and imaging, and their applications in pharmaceutical, security, and biological sciences.

Philip F. Taday received the B. Sc. (Hons.) degree in c.physics with specialization in laser physics from the University College of Swansea, Swansea, Wales, U.K., and the Ph.D. degree from the Department of Chemistry, University of Reading, Reading, Berkshire, U.K.

From November 1989 until July 2000, he was with the Central Laser Facility, Rutherford Appleton Laboratory, Didcot, U.K., as a Higher Scientific Officer, and became a Senior Scientific Officer in September 1999. Until 1992, he was engaged in research on the VULCAN Nd:glass laser program, and was also involved in the development of femtosecond tabletop laser system and novel tunable sources. In August 2000, he joined Toshiba Research Europe Ltd., Cambridge, U.K., where he was engaged in research on the development of terahertz sources. Currently, he is a Principal Scientist and Head of the Applications Group at TeraView Ltd., Cambridge. His current research interests include the application of terahertz radiation to real world problems.

Dr. Taday is a member of the Institute of Physics, U.K. 